\title{
Determinação de resíduos de agrotóxicos em Bombus spp. (Hymenoptera: Apidae) empregando o método Quechers modificado e LC-MS/MS e GC-MS/MS
}

Os polinizadores são fundamentais para a reprodução de diversas espécies de plantas com flores, atuando na transferência de grão de pólen de uma flor para outra. No entanto, suas atividades forrageiras os expõem em contato com diversos poluentes, incluindo os agrotóxicos. Pesquisas sobre efeitos adversos de agrotóxicos em abelhas do gênero Bombus são escasso no Brasil, e por serem insetos polinizadores, é importante realizar estudos que avaliem a suscetibilidade deles aos agrotóxicos utilizados na agricultura. Para a avaliação dos resíduos de agrotóxicos utilizados na agricultura para controle de pragas na cultura da soja no Rio Grande do Sul, foi empregado o método QuEChERS para a determinação de multirresíduo de agrotóxicos no corpo de abelhas. As abelhas do gênero Bombus utilizados na pesquisa foram coletados enquanto forrageavam em áreas de vegetação agrícola e urbana no Estado. O método QuEChERS para extração multirresíduo, envolve extração inicial em acetonitrila, etapa de partição após a adição de sal e uma etapa de limpeza utilizando extração em fase sólida dispersiva. No preparo para extração multirresíduo, envolve extração inicial em acetonitrila, etapa de partição após a adição de sal e uma etapa de limpeza utilizando extração em fase sólida dispersiva. No preparo
das amostras de Bombus spp. foi empregado o método QuEChERS para isolar os analitos das matrizes a serem estudadas. Na identificação e quantificação dos analitos foi utilizado um cromatógrafo líquido e gasoso acoplado a um detector de massas (LC-MS/MS e GC-MS/MS), para detecção de 119 compostos de diferentes classes, tais como, inseticidas e fungicidas. Dos 119 agrotóxicos analisados, apenas azoxistrobina, acetamiprido e metomil, apresentaram resíduos detectáveis nas amostras analisadas, no entanto, a azoxistrobina foi detectada na concentração abaixo do limite de quantificação do método (LOQ) para as amostras de B. morio coletados no município de Júlio de Castilho, enquanto que, o acetamiprido e metomil, obtiveram resíduos de agrotóxicos detectados acima do limite de quantificação do método também em amostras de B. morio, coletados nos municípios de Santiago e Capão do Cipó. Nos demais compostos (116), a concentração de agrotóxico obtida foi menor que o limite de deteç̧ão do método (LOD).

Palavras-chave: Polinizadores; Forrageamento; Contaminação; Cromatografia.

\section{Determination of pesticide residues in Bombus spp. (Hymenoptera: Apidae) using the modified Quechers method and LC-MS/MS and GC- $M S / M S$}

\begin{abstract}
Pollinators are essential for the reproduction of several species of flowering plants, acting on the transfer of pollen grain from one flower to another. However, their foraging activities as they explain in contact with various pollutants, including pesticides. Research on the adverse effects of pesticides on Bombus bees is scarce in Brazil, and because they are pollinating insects, it is important to carry out studies that assess their susceptibility to pesticides used in agriculture. For the evaluation of pesticide residues used in agriculture to control pests in soybean culture in Rio Grande do Sul, the QuEChERS method was used to determine multiresidue of pesticides in the body of bees. The Bombus bees used in the research were collected while foraging in areas of agricultural and urban vegetation in the State. The QuEChERS method for multi-residue extraction, involves initial extraction in acetonitrile, partitioning step after the addition of in areas of agricultural and urban vegetation in the State. The QuEChERS method for multi-residue extraction, involves initial extraction in acetonitrile, partitioning step after the addition of
salt and a cleaning step using dispersive solid phase extraction. Do not prepare Bombus spp. the QuEChERS method was developed to isolate the analytes from the matrices to be studied. In the identification and quantification of the analytes, a liquid and gas chromatograph coupled to a mass detector (LC-MS/ S and GC-MS/MS) was used to detect 119 compounds of different classes, such as insecticides and fungicides. Of the 119 promised pesticides, only azoxystrobin, acetamiprid and metomil, were detectable in the analyzed, but azoxystrobin was detected in the concentration below the method quantification limit (LOQ) for B. morio collected in the municipality of Júlio de Castilho, while acetamipride and metomil, obtained residues of pesticides detected above the limit of quantification of the method also in samples of B. morio, collected in the municipalities of Santiago and Capão do Cipó. In the other compounds (116), the concentration of pesticides obtained was below the limit of detection of the method (LOD).
\end{abstract}

Keywords: Pollinators; Foraging; Contamination; Chromatography.

Topic: Desenvolvimento, Sustentabilidade e Meio Ambiente

Reviewed anonymously in the process of blind peer.

Jocélia Gonçalves Silva (id

Universidade Federal de Santa Maria, Brasi

http://lattes.cnpq.br/2618132877154870

http://orcid.org/0000-0003-4459-9900

joceliags@hotmail.com

Joálisson Gonçalves Silva (iD

Universidade Federal da Paraíba, Brasil

http://lattes.cnpq.br/6079937299147425

http://orcid.org/0000-0001-5890-3833

joalissongs@yahoo.com.br

\section{Renato Zanella (iD)}

Universidade Federal de Santa Maria, Brasi

http://lattes.cnpq.br/2541865299438479

http://orcid.org/0000-0002-5971-1785

renato.zanella@ufsm.br

6

DOI: 10.6008/CBPC2179-6858.2021.006.0044
Received: 06/06/2021

Approved: 28/06/2021

Jerson Vanderlei Carús Guedes (iD

Universidade Federal de Santa Maria, Brasil

http://lattes.cnpq.br/0846418627719511

http://orcid.org/0000-0003-0652-3083

jerson.guedes@gmail.com
Referencing this:

SILVA, J. G.; SILVA, J. G.; ZANELLA, R.; GUEDES, J. V. C.. Determinação de resíduos de agrotóxicos em Bombus spp. (Hymenoptera: Apidae) empregando o método Quechers modificado e LC-MS/MS e GCMS/MS. Revista Ibero Americana de Ciências Ambientais, v.12, n.6, p.535-546, 2021. DOI: http://doi.org/10.6008/CBPC21796858.2021.006.0044 
Determinação de resíduos de agrotóxicos em Bombus spp. (Hymenoptera: Apidae) empregando o método Quechers modificado e LC-MS/MS e GC-MS/MS

\section{INTRODUÇÃO}

As abelhas são importantes polinizadores, sendo fundamentais para a reprodução de diversas espécies de plantas com flores, atuando na transferência de grão de pólen de uma flor para outra (BREEZE et al., 2011). No entanto, sua atividade forrageira os expõe em contato com diversos poluentes, incluindo os agrotóxicos (BADIOU-BÉNÉTEAU et al., 2013).

As populações de abelhas, principalmente Apis mellifera, vêm sendo submetidas a um declínio mundial, associado ao distúrbio do colapso das colmeias (CCD) e enfraquecimento da colônia (POTTS et al., 2010; VANENGELSDORP et al., 2009). Esse distúrbio proporciona uma perda drástica de abelhas adultas da colmeia sem qualquer evidência de mortalidade (VANENGELSDORP et al., 2009).

Suspeita-se que o declínio da população de abelhas resulta de uma combinação de vários fatores que poderia enfraquecê-las (PARADIS et al., 2014), entretanto, as utilizações indiscriminadas de agrotóxicos em cultivos agrícolas são apontadas como a principal causa da mortalidade de abelhas ( $A$. mellifera) e enfraquecimento de colônias, por pesquisadores e apicultores no Brasil e em vários países do mundo.

Os agrotóxicos são substâncias utilizadas com o propósito de prevenir, destruir ou controlar pragas agrícolas e com isso garantir alimento para uma população em constante crescimento. No entanto, sua utilização excessiva e sem critério tem causado impactos diretos e indiretos a saúde humana, animal e ao meio ambiente (FERRER et al., 2007). Muitos agrotóxicos usados na proteção de plantas, como o fipronil, imidaclopride, tiametoxam e deltametrina, são tóxicos para abelhas e são suspeitos de estarem envolvidos em perdas de colônias de Apis mellifera (VAN DER SLUIJS et al., 2013).

As abelhas são expostas a combinações de produtos químicos que podem provocar efeitos adversos em doses muito baixas (COLIN et al., 1998), sendo importante a quantificação simultânea de grande número de substâncias em baixos níveis de concentração (PARADIS et al., 2014). Pesquisas sobre efeitos adversos de agrotóxicos em abelhas do gênero Bombus são escassas no Brasil, e por serem insetos polinizadores, é importante realizar estudos que avaliem a suscetibilidade deles aos agrotóxicos comumente utilizados na agricultura.

O preparo de amostras para a determinação de níveis de agrotóxicos tem sido objeto de constantes estudos. A presença de interferentes que são extraídos junto com os analitos de interesse torna a detecção, identificação e quantificação de resíduos de agrotóxicos mais difíceis, especialmente em matrizes com diferentes constituintes, como o pólen apícola e abelhas. Buscando atender aos organismos regulamentadores, que estabelecem os níveis de resíduos permitidos, muitos métodos e técnicas têm sido utilizados no aperfeiçoamento do preparo de amostras para determinação de agrotóxicos, uma vez que a análise de resíduos de agrotóxicos em amostras de abelhas e pólen apícola é de extrema importância em termos de segurança e equilíbrio ambiental (SAIBT, 2017).

A presente pesquisa teve como objetivo determinar e quantificar a presença de resíduos de agrotóxicos em amostras de abelhas do gênero Bombus coletadas no Rio Grande do Sul, Brasil. Para esse estudo, foi empregado o método Quechers, uma técnica baseada em três etapas principais: (1) extração com 
Determinação de resíduos de agrotóxicos em Bombus spp. (Hymenoptera: Apidae) empregando o método Quechers modificado e LC-MS/MS e GC-MS/MS

acetonitrila; (2) partição promovida pela adição de sais, por exemplo: sulfato de magnésio (MgSO4) e cloreto de sódio $(\mathrm{NaCl})$ e (3) limpeza do extrato empregando a técnica denominada Extração em Fase Sólida Dispersiva (ZANELLA et al., 2015), com o objetivo de gerar extratos que possam ser analisados por Cromatografia Líquida e Cromatografia Gasosa acopladas a Espectrometria de Massas em Série (LC-MS/MS e GC-MS/MS), sendo possível analisar 119 agrotóxicos utilizados na agricultura para proteção de plantas.

\section{MATERIAIS E MÉTODOS}

\section{Coleta de material}

As coletas de Bombus spp. foram realizadas com rede entomológica no início do verão de 2019, período que se iniciam as aplicações de agrotóxicos para o controle de pragas na cultura da soja no Rio Grande do Sul. Quando necessário, utilizaram-se cabos adaptadores para facilitar a coleta de Bombus em árvores de grande porte. As coletas foram realizadas mediante autorizações do SISBIO (número de autorização: 59495-2) e SisGen (número de cadastro: A452394).

Os Bombus foram coletados em oito municípios do Rio Grande do Sul (Tabela 1) diretamente no recurso floral visitado durante o forrageamento, em áreas de vegetação agrícola e urbana. As plantas visitadas foram identificadas de acordo com informações registradas no site da Rede de Catálogos Polínicos - RCPol ${ }^{1}$ e Flora Brasil $2020^{2}$. Em cada ponto de coleta as coordenadas geográficas foram marcadas com auxílio de um GPS (Garmin).

Nos municípios amostrados, foram realizadas observações nos recursos florais existentes e quando detectada a presença de Bombus, estes eram coletados e transferidos para frascos plásticos. Foram coletados 10 exemplares de Bombus spp. por município amostrado. Os espécimes coletados foram mortos em ultrafreezer vertical $\left(-80^{\circ} \mathrm{C}\right)$ e transferidos para tubos tipo Falcon, separados de acordo com o município de coleta e espécies de Bombus coletadas. As espécies de Bombus foram etiquetadas e acondicionadas em freezer a $-20^{\circ} \mathrm{C}$ até a realização da análise de resíduos de agrotóxicos.

Tabela 1: Municípios da região central do Rio Grande do Sul com coletas de Bombus spp., recurso floral associado e data de amostragem.

\begin{tabular}{|c|c|c|c|c|}
\hline Espécie $(n=10)$ & Recurso Floral & Municípios & & Data \\
\hline Bombus morio & Senna macranthera & Santa Maria & $29^{\circ} 71^{\prime} \mathrm{S} 53^{\circ} 71^{\prime} \mathrm{W}$ & Mar 2019 \\
\hline Bombus morio & Jacaranda brasiliana & São Pedro do Sul & $29^{\circ} 63^{\prime} \mathrm{S} 54^{\circ} 18^{\prime} \mathrm{W}$ & Nov 2018 \\
\hline Bombus morio & Lagerstroemia indica & São Sepé & $30^{\circ} 15^{\prime} \mathrm{S} 53^{\circ} 58^{\prime} \mathrm{W}$ & Mar 2019 \\
\hline Bombus morio e & Lagerstroemia indica & Caçapava do Sul & $30^{\circ} 51^{\prime} \mathrm{S} 53^{\circ} 48^{\prime} \mathrm{W}$ & Mar 2019 \\
\hline Bombus pauloensis & Hibiscus syriacus & & & \\
\hline $\begin{array}{l}\text { Bombus morio e } \\
\text { Bombus pauloensis }\end{array}$ & Senna macranthera & Júlio de Castilhos & $29^{\circ} 25^{\prime} \mathrm{S} 53^{\circ} 66^{\prime} \mathrm{W}$ & Mar 2019 \\
\hline Bombus morio & Senna macranthera & Itaara & $29^{\circ} 54^{\prime} \mathrm{S} 53^{\circ} 75^{\prime} \mathrm{W}$ & Mar 2019 \\
\hline Bombus morio & Senna macranthera & Santiago & $29^{\circ} 20^{\prime} \mathrm{S} 54^{\circ} 85^{\prime} \mathrm{W}$ & Abr 2019 \\
\hline Bombus morio & Senna macranthera & Capão do Cipó & $28^{\circ} 92^{\prime} \mathrm{S} 54^{\circ} 55^{\prime} \mathrm{W}$ & Abr 2019 \\
\hline
\end{tabular}

\section{Método QuEChERS}

Foi empregado o método QuEChERS modificado visando a extração e determinação multirresíduo de

\footnotetext{
${ }^{1}$ http://chaves.rcpol.org.br/eco?

2 http://floradobrasil.jbrj.gov.br/reflora/listaBrasil/PrincipalUC/PrincipalUC.do\#CondicaoTaxonCP
} 
Determinação de resíduos de agrotóxicos em Bombus spp. (Hymenoptera: Apidae) empregando o método Quechers modificado e LC-MS/MS e

agrotóxicos em amostras de Bombus empregando LC-MS/MS e GC-MS/MS. A água foi purificada com sistema aMilli-Q (Millipore, Molsheim, França). Na sala de pesagem, pesou-se em balança analítica, $1 \mathrm{~g}$ da amostra de Bombus; os sais, $4 \mathrm{~g}$ de $\mathrm{MgSO}_{4} \mathrm{e} 1 \mathrm{~g} \mathrm{NaCl} ; 2 \mathrm{~mL}$ de extrato (solvente orgânico da amostra de Bombus), 300mg de $\mathrm{MgSO}_{4}, 250 \mathrm{mg}$ de $\mathrm{C} 18$ e 50mg de PSA. Na parte da extração adicionou-se a amostra, $5 \mathrm{~mL}$ de ACN (acetonitrila) e $5 \mathrm{~mL}$ de $\mathrm{H}_{2} \mathrm{O}$ ultra pura; 1 min no vórtex; em seguida acrescentou-se $4 \mathrm{~g}$ de $\mathrm{MgSO}_{4} \mathrm{e} 1 \mathrm{~g}$ de $\mathrm{NaCl}$; mais 1 min no vórtex; 8 min de centrifugação a 3400 rpm; colocou-se o sobrenadante por 1 h no freezer; acrescentou-se $2 \mathrm{~mL}$ de extrato e os sais; agitou-se por 1 min no vórtex para remover alguns interferentes; e centrifugou-se por $8 \mathrm{~min}$ por $6000 \mathrm{rpm}$ a $-10^{\circ} \mathrm{C}$. Posteriormente, procedeu-se a etapa de limpeza da amostra. Colocou-se 500uL de $\mathrm{H}_{2} \mathrm{O}$, 500uL da amostra de abelhas e $2 \mathrm{uL}$ de padrão interno (para controle do equipamento, pois pode não existir material na amostra analisada e com isso, pelo menos o padrão interno tem que ser observado). Este trabalho foi desenvolvido no Laboratório de Análises de Resíduos de Pesticidas (LARP) do Departamento de Química da Universidade Federal de Santa Maria (UFSM).

\section{Cromatografia}

Para determinação de resíduos de agrotóxicos foi utilizado a cromatografia gasosa e a cromatografia líquida. Na cromatografia gasosa, os componentes de uma amostra vaporizada são separados em consequência de sua interação entre uma fase móvel gasosa e uma fase estacionária líquida ou sólida contida em uma coluna, sendo esta aplicada à análise de compostos voláteis ou volatizáveis que sejam termicamente estáveis (COLLINS et al., 2009). Como a maioria dos compostos não são suficientemente voláteis para ser analisados por Cromatografia Gasosa, a Cromatografia Líquida de Alta Eficiência (HPLC, do inglês High Performance Liquid Chromatography) tem se destacado (SAIBT, 2017). Na HPLC o principal requisito para a análise é a solubilidade dos compostos na fase móvel (líquida) (COLLINS et al., 2009; HARRIS, 2012).

\section{Análise dos dados}

Os dados foram analisados de acordo com o método multirresíduo de extração para 119 agrotóxicos, com posterior análise por cromatografia gasosa e líquida de alta eficiência acoplada à espectrometria de massas em série (LC-MS/MS). O método proposto foi aplicado para análise de amostras de Bombus na região Central do Rio Grande do Sul, em localidades com confirmação e suspeita de mortalidade de Apis mellifera por contaminação de agrotóxicos, principalmente por fipronil.

\section{RESULTADOS}

Para os 119 agrotóxicos analisados pelo método QuEChERS modificado e LC-MS/MS e GC-MS/MS, apenas azoxistrobina, acetamiprido e metomil, apresentaram resíduos detectáveis nas amostras analisadas. Dessas 119 amostras analisadas, em 114, a concentração de agrotóxico obtida foi menor que o limite de detecção (LOD = Limite de detecção do método, do inglês, Limit of detection), em 1 amostra foi quantificado resíduo de agrotóxico, porém abaixo do limite de quantificação (LOQ = Limite de quantificação do método, 
Determinação de resíduos de agrotóxicos em Bombus spp. (Hymenoptera: Apidae) empregando o método Quechers modificado e LC-MS/MS e GC-MS/MS

do inglês, Limit of quantification), enquanto que em 4 amostras a concentração do agrotóxico foi detectada acima do limite de quantificação do método (Tabela 2).

Foi detectado resíduo de azoxistrobina na concentração menor que $0,005 \mathrm{mg} / \mathrm{kg}^{-1}$, sendo abaixo do limite de quantificação do método (LOQ), para a amostra de B. morio coletada no município de Júlio de Castilho-RS (Tabela 2). Para a amostra de B. morio coletada no município de Santiago-RS foi detectado resíduo de acetamiprido na concentração de $0,082 \mathrm{mg} / \mathrm{kg}^{-1}$, assim como também foi detectado resíduo do mesmo agrotóxico na concentração de $0,013 \mathrm{mg} / \mathrm{kg}^{-1}$ para a amostra de $B$. morio coletada no município de Capão do Cipó-RS. Nas amostras de B. morio coletadas no município de Santiago-RS e Capão do Cipó-RS, foi detectado resíduo de metomil na concentração de $0,058 \mathrm{mg} / \mathrm{kg}^{-1} \mathrm{e} 0,019 \mathrm{mg} / \mathrm{kg}^{-1}$, respectivamente (Tabela 2).

Apesar de ter sido detectada a presença de resíduos de agrotóxicos apenas em B. morio, não é possível afirmar que esta espécie seja mais susceptível aos resíduos desses produtos que B. pauloensis, uma vez que, a espécie $B$. morio está presente em maior quantidade e mais distribuída no Estado que a espécie B. pauloensis, e com isso mais exposta aos poluentes, principalmente os agrotóxicos, durante sua atividade de forrageamento (coleta de pólen e néctar). Mais estudos sobre a presença de resíduos de agrotóxicos em Bombus no Rio Grande do Sul são necessários, visto que nessa pesquisa foi abordado a coleta de Bombus em apenas 8 municípios. O primeiro passo foi dado, mais é importante dá continuidade a pesquisa para se ter mais embasamentos para afirmar ou negar a influência dos agrotóxicos no desparecimento de algumas espécies de Bombus no Estado.

Tabela 2: Determinação de resíduos de agrotóxicos em Bombus morio e Bombus pauloensis em 8 municípios do Rio Grande do Sul, Brasil.

\begin{tabular}{|c|c|c|c|c|c|c|c|c|c|c|c|c|}
\hline \multicolumn{3}{|l|}{ Agrotóxico } & $\begin{array}{l}\text { Júlio de } \\
\text { Castilhos } \\
\text { Bombus } \\
\text { morio }\end{array}$ & $\begin{array}{l}\text { Júlio de } \\
\text { Castilhos } \\
\text { Bombus } \\
\text { pauloensis }\end{array}$ & \multirow{2}{*}{$\begin{array}{l}\text { Santa } \\
\text { Maria } \\
\text { Bombus } \\
\text { morio }\end{array}$} & \multirow{2}{*}{$\begin{array}{l}\text { Caçapava } \\
\text { do Sul } \\
\text { Bombus } \\
\text { morio }\end{array}$} & \multirow{2}{*}{$\begin{array}{l}\text { Caçapava } \\
\text { do Sul } \\
\text { Bombus } \\
\text { pauloensis }\end{array}$} & \multirow{2}{*}{$\begin{array}{l}\text { São } \\
\text { Sepé } \\
\text { Bombus } \\
\text { morio }\end{array}$} & \multirow{2}{*}{$\begin{array}{l}\text { Itaara } \\
\text { Bombus } \\
\text { morio }\end{array}$} & \multirow{2}{*}{$\begin{array}{l}\text { Santiago } \\
\text { Bombus } \\
\text { morio }\end{array}$} & \multirow{2}{*}{$\begin{array}{l}\text { Capão } \\
\text { do Cipó } \\
\text { Bombus } \\
\text { morio }\end{array}$} & \multirow{2}{*}{$\begin{array}{l}\text { São } \\
\text { Pedro } \\
\text { do Sul } \\
\text { Bombus } \\
\text { morio } \\
\end{array}$} \\
\hline $\begin{array}{l}\text { Compostos } \\
\text { Analisados }\end{array}$ & $\begin{array}{l}\text { LOD } \\
(\mathrm{mg} \\
\left.\mathrm{kg}^{-1}\right) \\
\end{array}$ & $\begin{array}{l}\text { LOQ } \\
(\mathrm{mg} \\
\left.\mathrm{kg}^{-1}\right) \\
\end{array}$ & $\begin{array}{l}\text { Concentra } \\
\left(\mathrm{mg} \mathrm{kg}^{-1}\right)\end{array}$ & & & & & & & & & \\
\hline Acetamiprido & 0,002 & 0,005 & n.d. & n.d. & n.d. & n.d. & n.d. & n.d. & n.d. & 0,082 & 0,013 & n.d. \\
\hline Aldicarbe & 0,002 & 0,005 & n.d. & n.d. & n.d. & n.d. & n.d. & n.d. & n.d. & n.d. & n.d. & n.d. \\
\hline Ametrina & 0,002 & 0,005 & n.d. & n.d. & n.d. & n.d. & n.d. & n.d. & n.d. & n.d. & n.d. & n.d. \\
\hline Atrazina & 0,002 & 0,005 & n.d. & n.d. & n.d. & n.d. & n.d. & n.d. & n.d. & n.d. & n.d. & n.d. \\
\hline Azaconazol & 0,002 & 0,005 & n.d. & n.d. & n.d. & n.d. & n.d. & n.d. & n.d. & n.d. & n.d. & n.d. \\
\hline Azametifós & 0,002 & 0,005 & n.d. & n.d. & n.d. & n.d. & n.d. & n.d. & n.d. & n.d. & n.d. & n.d. \\
\hline Azinfós-etílico & 0,008 & 0,025 & n.d. & n.d. & n.d. & n.d. & n.d. & n.d. & n.d. & n.d. & n.d. & n.d. \\
\hline Azinfós-metílico & 0,002 & 0,005 & n.d. & n.d. & n.d. & n.d. & n.d. & n.d. & n.d. & n.d. & n.d. & n.d. \\
\hline Azoxistrobina & 0,002 & 0,005 & $<\mathrm{LOQ}$ & n.d. & n.d. & n.d. & n.d. & n.d. & n.d. & n.d. & n.d. & n.d. \\
\hline Bitertanol & 0,003 & 0,010 & n.d. & n.d. & n.d. & n.d. & n.d. & n.d. & n.d. & n.d. & n.d. & n.d. \\
\hline Boscalida & 0,002 & 0,005 & n.d. & n.d. & n.d. & n.d. & n.d. & n.d. & n.d. & n.d. & n.d. & n.d. \\
\hline $\begin{array}{l}\text { Bromofós- } \\
\text { metílico }\end{array}$ & 0,002 & 0,005 & n.d. & n.d. & n.d. & n.d. & n.d. & n.d. & n.d. & n.d. & n.d. & n.d. \\
\hline Bromuconazol & 0,003 & 0,010 & n.d. & n.d. & n.d. & n.d. & n.d. & n.d. & n.d. & n.d. & n.d. & n.d. \\
\hline Buprofenzina & 0,002 & 0,005 & n.d. & n.d. & n.d. & n.d. & n.d. & n.d. & n.d. & n.d. & n.d. & n.d. \\
\hline \begin{tabular}{ll|}
$\begin{array}{l}\text { Butóxido } \\
\text { piperolina }\end{array}$ & de \\
\end{tabular} & 0,002 & 0,005 & n.d. & n.d. & n.d. & n.d. & n.d. & n.d. & n.d. & n.d. & n.d. & n.d. \\
\hline Carbaril & 0,002 & 0,005 & n.d. & n.d. & n.d. & n.d. & n.d. & n.d. & n.d. & n.d. & n.d. & n.d. \\
\hline Carbofurano & 0,002 & 0,005 & n.d. & n.d. & n.d. & n.d. & n.d. & n.d. & n.d. & n.d. & n.d. & n.d. \\
\hline $\begin{array}{l}\text { Carbofurano-3- } \\
\text { hidróxido }\end{array}$ & 0,002 & 0,005 & n.d. & n.d. & n.d. & n.d. & n.d. & n.d. & n.d. & n.d. & n.d. & n.d. \\
\hline Carboxina & 0,002 & 0,005 & n.d. & n.d. & n.d. & n.d. & n.d. & n.d. & n.d. & n.d. & n.d. & n.d. \\
\hline Cialofope Butílico & 0,002 & 0,005 & n.d. & n.d. & n.d. & n.d. & n.d. & n.d. & n.d. & n.d. & n.d. & n.d. \\
\hline Cianazina & 0,008 & 0,025 & n.d. & n.d. & n.d. & n.d. & n.d. & n.d. & n.d. & n.d. & n.d. & n.d. \\
\hline Ciazofamida & 0,002 & 0,005 & n.d. & n.d. & n.d. & n.d. & n.d. & n.d. & n.d. & n.d. & n.d. & n.d. \\
\hline Cimoxanil & 0,002 & 0,005 & n.d. & n.d. & n.d. & n.d. & n.d. & n.d. & n.d. & n.d. & n.d. & n.d. \\
\hline Clomazona & 0,002 & 0,005 & n.d. & n.d. & n.d. & n.d. & n.d. & n.d. & n.d. & n.d. & n.d. & n.d. \\
\hline
\end{tabular}




\begin{tabular}{|c|c|c|c|c|c|c|c|c|c|c|c|c|}
\hline Clorantraniliprole & 0,002 & 0,005 & n.d. & n.d. & n.d. & n.d. & n.d. & n.d. & n.d. & n.d. & n.d. & n.d. \\
\hline Clorpirifós-etílico & 0,002 & 0,005 & n.d. & n.d. & n.d. & n.d. & n.d. & n.d. & n.d. & n.d. & n.d. & n.d. \\
\hline $\begin{array}{l}\text { Clorpirifós- } \\
\text { metílico }\end{array}$ & 0,002 & 0,005 & n.d. & n.d. & n.d. & n.d. & n.d. & n.d. & n.d. & n.d. & n.d. & n.d. \\
\hline Clorprofam & 0,003 & 0,010 & n.d. & n.d. & n.d. & n.d. & n.d. & n.d. & n.d. & n.d. & n.d. & n.d. \\
\hline Clotianidina & 0,008 & 0,025 & n.d. & n.d. & n.d. & n.d. & n.d. & n.d. & n.d. & n.d. & n.d. & n.d. \\
\hline $\begin{array}{l}\text { Cresoxim- } \\
\text { metílico }\end{array}$ & 0,003 & 0,010 & n.d. & n.d. & n.d. & n.d. & n.d. & n.d. & n.d. & n.d. & n.d. & n.d. \\
\hline Deltametrina & 0,008 & 0,025 & n.d. & n.d. & n.d. & n.d. & n.d. & n.d. & n.d. & n.d. & n.d. & n.d. \\
\hline $\begin{array}{l}\text { Demeton-S- } \\
\text { metil-sulfona }\end{array}$ & 0,003 & 0,010 & n.d. & n.d. & n.d. & n.d. & n.d. & n.d. & n.d. & n.d. & n.d. & n.d. \\
\hline Diazinona & 0,002 & 0,005 & n.d. & n.d. & n.d. & n.d. & n.d. & n.d. & n.d. & n.d. & n.d. & n.d. \\
\hline Dicrotofós & 0,003 & 0,010 & n.d. & n.d. & n.d. & n.d. & n.d. & n.d. & n.d. & n.d. & n.d. & n.d. \\
\hline Difenoconazol & 0,002 & 0,005 & n.d. & n.d. & n.d. & n.d. & n.d. & n.d. & n.d. & n.d. & n.d. & n.d. \\
\hline Dimetoato & 0,002 & 0,005 & n.d. & n.d. & n.d. & n.d. & n.d. & n.d. & n.d. & n.d. & n.d. & n.d. \\
\hline Dimoxistrobina & 0,002 & 0,005 & n.d. & n.d. & n.d. & n.d. & n.d. & n.d. & n.d. & n.d. & n.d. & n.d. \\
\hline Diniconazol & 0,003 & 0,010 & n.d. & n.d. & n.d. & n.d. & n.d. & n.d. & n.d. & n.d. & n.d. & n.d. \\
\hline Diurom & 0,002 & 0,005 & n.d. & n.d. & n.d. & n.d. & n.d. & n.d. & n.d. & n.d. & n.d. & n.d. \\
\hline EPN & 0,002 & 0,010 & n.d. & n.d. & n.d. & n.d. & n.d. & n.d. & n.d. & n.d. & n.d. & n.d. \\
\hline Epoxiconazol & 0,002 & 0,005 & n.d. & n.d. & n.d. & n.d. & n.d. & n.d. & n.d. & n.d. & n.d. & n.d. \\
\hline Etiofencarbe & 0,002 & 0,005 & n.d. & n.d. & n.d. & n.d. & n.d. & n.d. & n.d. & n.d. & n.d. & n.d. \\
\hline Etoprofós & 0,003 & 0,010 & n.d. & n.d. & n.d. & n.d. & n.d. & n.d. & n.d. & n.d. & n.d. & n.d. \\
\hline Famoxadona & 0,003 & 0,010 & n.d. & n.d. & n.d. & n.d. & n.d. & n.d. & n.d. & n.d. & n.d. & n.d. \\
\hline Fempiroximato & 0,002 & 0,005 & n.d. & n.d. & n.d. & n.d. & n.d. & n.d. & n.d. & n.d. & n.d. & n.d. \\
\hline Fenamidona & 0,002 & 0,005 & n.d. & n.d. & n.d. & n.d. & n.d. & n.d. & n.d. & n.d. & n.d. & n.d. \\
\hline Fenamifós & 0,002 & 0,005 & n.d. & n.d. & n.d. & n.d. & n.d. & n.d. & n.d. & n.d. & n.d. & n.d. \\
\hline Fenarimol & 0,002 & 0,005 & n.d. & n.d. & n.d. & n.d. & n.d. & n.d. & n.d. & n.d. & n.d. & n.d. \\
\hline Fenhexamida & 0,003 & 0,010 & n.d. & n.d. & n.d. & n.d. & n.d. & n.d. & n.d. & n.d. & n.d. & n.d. \\
\hline Fenoxicarbe & 0,002 & 0,005 & n.d. & n.d. & n.d. & n.d. & n.d. & n.d. & n.d. & n.d. & n.d. & n.d. \\
\hline Fentiona & 0,002 & 0,005 & n.d. & n.d. & n.d. & n.d. & n.d. & n.d. & n.d. & n.d. & n.d. & n.d. \\
\hline Fipronil & 0,008 & 0,025 & n.d. & n.d. & n.d. & n.d. & n.d. & n.d. & n.d. & n.d. & n.d. & n.d. \\
\hline $\begin{array}{l}\text { Fluasifope-P- } \\
\text { butílico }\end{array}$ & 0,002 & 0,005 & n.d. & n.d. & n.d. & n.d. & n.d. & n.d. & n.d. & n.d. & n.d. & n.d. \\
\hline Fluquinconazol & 0,002 & 0,005 & n.d. & n.d. & n.d. & n.d. & n.d. & n.d. & n.d. & n.d. & n.d. & n.d. \\
\hline Flusilazol & 0,002 & 0,005 & n.d. & n.d. & n.d. & n.d. & n.d. & n.d. & n.d. & n.d. & n.d. & n.d. \\
\hline Flutolanil & 0,002 & 0,005 & n.d. & n.d. & n.d. & n.d. & n.d. & n.d. & n.d. & n.d. & n.d. & n.d. \\
\hline Flutriatol & 0,008 & 0,025 & n.d. & n.d. & n.d. & n.d. & n.d. & n.d. & n.d. & n.d. & n.d. & n.d. \\
\hline Forato & 0,003 & 0,010 & n.d. & n.d. & n.d. & n.d. & n.d. & n.d. & n.d. & n.d. & n.d. & n.d. \\
\hline Fosalona & 0,002 & 0,005 & n.d. & n.d. & n.d. & n.d. & n.d. & n.d. & n.d. & n.d. & n.d. & n.d. \\
\hline Fosmete & 0,002 & 0,005 & n.d. & n.d. & n.d. & n.d. & n.d. & n.d. & n.d. & n.d. & n.d. & n.d. \\
\hline Furatiocarbe & 0,002 & 0,005 & n.d. & n.d. & n.d. & n.d. & n.d. & n.d. & n.d. & n.d. & n.d. & n.d. \\
\hline Hexitiazoxi & 0,002 & 0,005 & n.d. & n.d. & n.d. & n.d. & n.d. & n.d. & n.d. & n.d. & n.d. & n.d. \\
\hline Iprovalicarbe & 0,002 & 0,005 & n.d. & n.d. & n.d. & n.d. & n.d. & n.d. & n.d. & n.d. & n.d. & n.d. \\
\hline Malationa & 0,002 & 0,005 & n.d. & n.d. & n.d. & n.d. & n.d. & n.d. & n.d. & n.d. & n.d. & n.d. \\
\hline Mecarbam & 0,003 & 0,010 & n.d. & n.d. & n.d. & n.d. & n.d. & n.d. & n.d. & n.d. & n.d. & n.d. \\
\hline Mefosfolan & 0,002 & 0,005 & n.d. & n.d. & n.d. & n.d. & n.d. & n.d. & n.d. & n.d. & n.d. & n.d. \\
\hline Mepronil & 0,002 & 0,005 & n.d. & n.d. & n.d. & n.d. & n.d. & n.d. & n.d. & n.d. & n.d. & n.d. \\
\hline Metalaxil & 0,002 & 0,005 & n.d. & n.d. & n.d. & n.d. & n.d. & n.d. & n.d. & n.d. & n.d. & n.d. \\
\hline Metconazol & 0,002 & 0,005 & n.d. & n.d. & n.d. & n.d. & n.d. & n.d. & n.d. & n.d. & n.d. & n.d. \\
\hline Metidationa & 0,008 & 0,025 & n.d. & n.d. & n.d. & n.d. & n.d. & n.d. & n.d. & n.d. & n.d. & n.d. \\
\hline Metiocarbe & 0,002 & 0,005 & n.d. & n.d. & n.d. & n.d. & n.d. & n.d. & n.d. & n.d. & n.d. & n.d. \\
\hline $\begin{array}{l}\text { Metiocarbe- } \\
\text { sulfona }\end{array}$ & 0,003 & 0,010 & n.d. & n.d. & n.d. & n.d. & n.d. & n.d. & n.d. & n.d. & n.d. & n.d. \\
\hline $\begin{array}{l}\text { Metiocarbe- } \\
\text { sulfóxido }\end{array}$ & 0,002 & 0,005 & n.d. & n.d. & n.d. & n.d. & n.d. & n.d. & n.d. & n.d. & n.d. & n.d. \\
\hline Metomil & 0,003 & 0,010 & n.d. & n.d. & n.d. & n.d. & n.d. & n.d. & n.d. & 0,058 & 0,019 & n.d. \\
\hline Metoxifenozida & 0,002 & 0,005 & n.d. & n.d. & n.d. & n.d. & n.d. & n.d. & n.d. & n.d. & n.d. & n.d. \\
\hline Mevinfós & 0,008 & 0,025 & n.d. & n.d. & n.d. & n.d. & n.d. & n.d. & n.d. & n.d. & n.d. & n.d. \\
\hline Miclobutanil & 0,002 & 0,005 & n.d. & n.d. & n.d. & n.d. & n.d. & n.d. & n.d. & n.d. & n.d. & n.d. \\
\hline Molinato & 0,002 & 0,005 & n.d. & n.d. & n.d. & n.d. & n.d. & n.d. & n.d. & n.d. & n.d. & n.d. \\
\hline Monocrotofós & 0,008 & 0,025 & n.d. & n.d. & n.d. & n.d. & n.d. & n.d. & n.d. & n.d. & n.d. & n.d. \\
\hline Monolinurom & 0,002 & 0,005 & n.d. & n.d. & n.d. & n.d. & n.d. & n.d. & n.d. & n.d. & n.d. & n.d. \\
\hline Nuarimol & 0,008 & 0,025 & n.d. & n.d. & n.d. & n.d. & n.d. & n.d. & n.d. & n.d. & n.d. & n.d. \\
\hline Oxadixil & 0,008 & 0,0025 & n.d. & n.d. & n.d. & n.d. & n.d. & n.d. & n.d. & n.d. & n.d. & n.d. \\
\hline Oxamil & 0,003 & 0,010 & n.d. & n.d. & n.d. & n.d. & n.d. & n.d. & n.d. & n.d. & n.d. & n.d. \\
\hline Oxifluorfem & 0,002 & 0,005 & n.d. & n.d. & n.d. & n.d. & n.d. & n.d. & n.d. & n.d. & n.d. & n.d. \\
\hline Paraoxom-etílico & 0,002 & 0,005 & n.d. & n.d. & n.d. & n.d. & n.d. & n.d. & n.d. & n.d. & n.d. & n.d. \\
\hline Parationa-etílica & 0,008 & 0,025 & n.d. & n.d. & n.d. & n.d. & n.d. & n.d. & n.d. & n.d. & n.d. & n.d. \\
\hline Pencicurom & 0,002 & 0,005 & n.d. & n.d. & n.d. & n.d. & n.d. & n.d. & n.d. & n.d. & n.d. & n.d. \\
\hline Penconazol & 0,002 & 0,005 & n.d. & n.d. & n.d. & n.d. & n.d. & n.d. & n.d. & n.d. & n.d. & n.d. \\
\hline Pirazofós & 0,002 & 0,005 & n.d. & n.d. & n.d. & n.d. & n.d. & n.d. & n.d. & n.d. & n.d. & n.d. \\
\hline Piridabem & 0,002 & 0,005 & n.d. & n.d. & n.d. & n.d. & n.d. & n.d. & n.d. & n.d. & n.d. & n.d. \\
\hline Piridafentiona & 0,002 & 0,005 & n.d. & n.d. & n.d. & n.d. & n.d. & n.d. & n.d. & n.d. & n.d. & n.d. \\
\hline Pirimetanil & 0,002 & 0,005 & n.d. & n.d. & n.d. & n.d. & n.d. & n.d. & n.d. & n.d. & n.d. & n.d. \\
\hline Pirimicarbe & 0,002 & 0,005 & n.d. & n.d. & n.d. & n.d. & n.d. & n.d. & n.d. & n.d. & n.d. & n.d. \\
\hline Pirimifós-etílico & 0,002 & 0,005 & n.d. & n.d. & n.d. & n.d. & n.d. & n.d. & n.d. & n.d. & n.d. & n.d. \\
\hline Pirimifós-metílico & 0,002 & 0,005 & n.d. & n.d. & n.d. & n.d. & n.d. & n.d. & n.d. & n.d. & n.d. & n.d. \\
\hline Piriproxifeno & 0,002 & 0,005 & n.d. & n.d. & n.d. & n.d. & n.d. & n.d. & n.d. & n.d. & n.d. & n.d. \\
\hline Profenofós & 0,002 & 0,005 & n.d. & n.d. & n.d. & n.d. & n.d. & n.d. & n.d. & n.d. & n.d. & n.d. \\
\hline Prometrina & 0,002 & 0,005 & n.d. & n.d. & n.d. & n.d. & n.d. & n.d. & n.d. & n.d. & n.d. & n.d. \\
\hline Propanil & 0,008 & 0,025 & n.d. & n.d. & n.d. & n.d. & n.d. & n.d. & n.d. & n.d. & n.d. & n.d. \\
\hline
\end{tabular}


Determinação de resíduos de agrotóxicos em Bombus spp. (Hymenoptera: Apidae) empregando o método Quechers modificado e LC-MS/MS e GC-MS/MS

SILVA, J. G.; SILVA, J. G.; ZANELLA, R.; GUEDES, J. V. C.

\begin{tabular}{|c|c|c|c|c|c|c|c|c|c|c|c|c|}
\hline Propiconazol & 0,002 & 0,005 & n.d. & n.d. & n.d. & n.d. & n.d. & n.d. & n.d. & n.d. & n.d. & n.d. \\
\hline Propoxur & 0,002 & 0,005 & n.d. & n.d. & n.d. & n.d. & n.d. & n.d. & n.d. & n.d. & n.d. & n.d. \\
\hline Quinalfós & 0,002 & 0,005 & n.d. & n.d. & n.d. & n.d. & n.d. & n.d. & n.d. & n.d. & n.d. & n.d. \\
\hline $\begin{array}{l}\text { Quizalofope-P- } \\
\text { etílico }\end{array}$ & 0,002 & 0,005 & n.d. & n.d. & n.d. & n.d. & n.d. & n.d. & n.d. & n.d. & n.d. & n.d. \\
\hline Simazina & 0,008 & 0,025 & n.d. & n.d. & n.d. & n.d. & n.d. & n.d. & n.d. & n.d. & n.d. & n.d. \\
\hline Tebuconazol & 0,003 & 0,010 & n.d. & n.d. & n.d. & n.d. & n.d. & n.d. & n.d. & n.d. & n.d. & n.d. \\
\hline Tebufenozida & 0,002 & 0,005 & n.d. & n.d. & n.d. & n.d. & n.d. & n.d. & n.d. & n.d. & n.d. & n.d. \\
\hline Tebufempirade & 0,002 & 0,005 & n.d. & n.d. & n.d. & n.d. & n.d. & n.d. & n.d. & n.d. & n.d. & n.d. \\
\hline Terbutilazina & 0,002 & 0,005 & n.d. & n.d. & n.d. & n.d. & n.d. & n.d. & n.d. & n.d. & n.d. & n.d. \\
\hline Tetraconazol & 0,002 & 0,005 & n.d. & n.d. & n.d. & n.d. & n.d. & n.d. & n.d. & n.d. & n.d. & n.d. \\
\hline Tiacloprido & 0,002 & 0,005 & n.d. & n.d. & n.d. & n.d. & n.d. & n.d. & n.d. & n.d. & n.d. & n.d. \\
\hline Tiametoxam & 0,008 & 0,025 & n.d. & n.d. & n.d. & n.d. & n.d. & n.d. & n.d. & n.d. & n.d. & n.d. \\
\hline Tiobencarbe & 0,002 & 0,005 & n.d. & n.d. & n.d. & n.d. & n.d. & n.d. & n.d. & n.d. & n.d. & n.d. \\
\hline Tiodicarbe & 0,002 & 0,005 & n.d. & n.d. & n.d. & n.d. & n.d. & n.d. & n.d. & n.d. & n.d. & n.d. \\
\hline $\begin{array}{l}\text { Tiofanato- } \\
\text { metílico }\end{array}$ & 0,002 & 0,005 & n.d. & n.d. & n.d. & n.d. & n.d. & n.d. & n.d. & n.d. & n.d. & n.d. \\
\hline Tolcofós-metílico & 0,008 & 0,025 & n.d. & n.d. & n.d. & n.d. & n.d. & n.d. & n.d. & n.d. & n.d. & n.d. \\
\hline Triazofós & 0,002 & 0,005 & n.d. & n.d. & n.d. & n.d. & n.d. & n.d. & n.d. & n.d. & n.d. & n.d. \\
\hline Triciclazol & 0,003 & 0,010 & n.d. & n.d. & n.d. & n.d. & n.d. & n.d. & n.d. & n.d. & n.d. & n.d. \\
\hline Triflumurom & 0,002 & 0,005 & n.d. & n.d. & n.d. & n.d. & n.d. & n.d. & n.d. & n.d. & n.d. & n.d. \\
\hline Vamidotiona & 0,003 & 0,010 & n.d. & n.d. & n.d. & n.d. & n.d. & n.d. & n.d. & n.d. & n.d. & n.d. \\
\hline
\end{tabular}

LOD = Limite de detecção do método (do inglês, Limit of detection)

$\mathrm{LOQ}=$ Limite de quantificação do método (do inglês, Limit of quantification)

n.d = Não detectado, ou seja, menor que o limite de detecção.

\section{DISCUSSÃO}

O uso dos agrotóxicos é importante para a agricultura principalmente quando se trata de aumento de produtividade, garantindo o controle de insetos pragas e doenças (VEIGA, 2006) que comprometem o desenvolvimento e produção desejada das culturas. Entretanto, muitas dessas práticas agrícolas ocorrem sem o manejo correto colocando em risco a manutenção da biodiversidade de abelhas e perdas de espécies como descrito para a Apis mellifera no fenômeno denominado Colony Collapse Disorder (CCD) relatado nos Estados Unidos, onde apicultores chegaram a perder 90\% de suas colmeias (ELLIS, 2007).

A Desordem do Colapso das Colônias (no português) está associada ao desaparecimento de abelhas operárias forrageiras, encarregadas de coletar o néctar e o pólen nas flores, e que não retornam às colmeias. Entretanto, as causas ainda não estão definidas, embora esteja associada à intoxicação de abelhas por agrotóxicos, que são cada vez mais utilizados na agricultura moderna. Na pesquisa desenvolvida por SABIT (2017), o método proposto para deteç̧ão de resíduos de agrotóxicos aplicado em amostras de pólen apícola e de abelhas A. mellifera, obtidas de diferentes regiões do estado do Rio Grande do Sul, todas as amostras apresentaram resíduos de agrotóxicos, porém abaixo do limite máximo estabelecido pela legislação Europeia.

Segundo Malaspina et al. (2008), os inseticidas podem afetar as abelhas principalmente por três modos de intoxicação: a) contato; b) ingestão e c) fumigação e, seus efeitos variam de morte causada por toxicidade aguda e efeitos a longo prazo provocando danos no funcionamento da colônia e diminuição da longevidade dos indivíduos. Na Tabela 3 foi observada a toxicidade de contato e a toxicidade oral de alguns agrotóxicos para as abelhas, medida pela dose letal mediana ( $\mathrm{LD}_{50}$, do inglês Lethal Dose) (BAYO et al., 2016). $\mathrm{LD}_{50}$ corresponde à dose necessária de uma dada substância para matar 50\% de uma população. 
Determinação de resíduos de agrotóxicos em Bombus spp. (Hymenoptera: Apidae) empregando o método Quechers modificado e LC-MS/MS e GC-MS/MS

SILVA, J. G.; SILVA, J. G.; ZANELLA, R.; GUEDES, J. V. C.

Tabela 3: Classe, agrotóxico e suas respectivas toxicidades por contato e oral para as abelhas.

\begin{tabular}{llll}
\hline Classe & Agrotóxicos & $\begin{array}{l}\mathbf{L D}_{50} \text { Contato } \\
\left(\boldsymbol{\mu g} \mathbf{~ k g}^{-1}\right)\end{array}$ & $\begin{array}{l}\mathbf{L D}_{\mathbf{5 0}} \text { Oral } \\
\left(\boldsymbol{\mu g} \mathbf{~ k g}^{-1}\right)\end{array}$ \\
\hline Inseticidas & Carbofurano & 0,16 & 0,24 \\
& Clorpirifós & 0,072 & 0,004 \\
& Clotianidina & 0,039 & 0,21 \\
& Diazinona & 0,38 & 0,001 \\
& Fipronil & 0,007 & 0,013 \\
& Fenpiroximato & 11 & 45 \\
\hline Imidacloprido & 0,061 & 25 \\
& Tau-Fluvalinato & 8,7 & 166 \\
& Azoxistrobina & 200 & 34 \\
& Boscalida & 200 & 77 \\
& Carbendazim & 50 & 8 \\
\hline
\end{tabular}

Fonte: Bayo et al. (2016).

Na tabela acima, dos agrotóxicos presentes nas amostras de Bombus spp. analisadas na pesquisa, está listado apenas o fungicida Azoxistrobina, no entanto o inseticida Acetamiprido e o inseticida e acaricida Metomil são amplamente utilizados na agricultura para controle dos principais insetos-praga e doenças de diversas culturas de importância agrícola no Rio Grande do Sul.

Em pesquisa desenvolvida por Gradish et al. (2012), B. impatiens foi suscetível a exposição tópica de todos os inseticidas testados, exceto Flubendiamida, que não causou mortalidade na dosagem de até 5000 $\mathrm{mg}$ a.i./L, aproximadamente quatro vezes a taxa de aplicação recomendada que é de $525 \mathrm{mg}$ a.i./L, e não representa perigo com contato direto para B. impatiens. Enquanto que, com base nos valores $\mathrm{LC}_{50}$, Deltametrina foi o agrotóxico mais tóxico, sendo 6,8, 2,2 e 1,7 vezes mais suscetíveis que o Spinosad, Spinetoram e Phosmet, respectivamente.

\section{Azoxistrobina}

A Azoxistrobina (C22H17N3O5) é um composto de metoxiacrilato e um fungicida pertencente ao grupo químico estrobilurinas. Sua base de formulação é de compostos antifúngicos de ocorrência natural em cogumelos que se desenvolvem em madeira em decomposição (MINISTRY OF HEALTH, 2019); trata-se de um fungicida de pós-emergência, sistêmico e de amplo espectro, com atividade contra os quatro maiores grupos de fungos patogênicos (ascomicetos, basidiomicetos, deutoromicetos e oomicetos) (MINISTRY OF HEALTH, 2019). Sua ação é de inibição da respiração mitocondrial, aderindo ao citocromo "b", o que impede a passagem de elétrons dos citocromos " $\mathrm{b}$ " para os " $\mathrm{c}$ ", e consequentemente interrompe a produção de energia. O resultado é a morte dos microrganismos (BARTLETT et al., 2002).

Este composto é um dos fungicidas mais comercializados em todo o mundo, como substituição aos fungicidas triazólicos; no entanto enfrentam também sérios questionamentos (MINISTRY OF HEALTH, 2019). No Brasil tem uso autorizado pela ANVISA para: (i) aplicação foliar nas culturas de abacate, abóbora, abobrinha, alface, algodão, alho, amendoim, arroz, aveia, banana, batata, begônia, berinjela, beterraba, café, caju, caqui, cana-de-açúcar, chalota, cebola, cenoura, centeio, cevada, citros, couve-flor, crisântemo, ervilha, eucalipto, feijão, figo, gérbera, girassol, goiaba, kalanchoe, mamão, manga, maracujá, melancia, melão, milho, milheto, morango, nectarina, pepino, pêssego, pimentão, soja, sorgo, tomate, trigo, triticale e uva; (ii) 
Determinação de resíduos de agrotóxicos em Bombus spp. (Hymenoptera: Apidae) empregando o método Quechers modificado e LC-MS/MS e

aplicação em sementes de algodão; (iii) e tratamento industrial de propágulos vegetativos (mudas) antes do plantio na cultura de cana-de-açúcar.

A azoxistrobina aparece na lista de vendas da ANVISA a partir de 2012, oscilando entre a 27ạ posição em 2012, 17ạ em 2015 (ano em que se registrou a maior venda: 3643 toneladas) e 27ạ em 2017. Destaque para região Centro Oeste com 40\% das vendas de 2012 a 2017 e, entre os estados, o MT, com 24\% (IBAMA, 2018). A azoxistrobina possui baixa solubilidade (6-10 mg/L) (MINISTRY OF HEALTH, 2019) e não é volátil (PPDB, 2019). No solo, apresenta mobilidade moderada à baixa (MINISTRY OF HEALTH, 2019). Porém, exibe degradação muito lenta no solo e em sedimentos - tempo de meia-vida no solo de 279 dias, com $24-42 \%$ do composto remanescente após 360 dias; em condições anaeróbias observou-se meia-vida de 181 dias e 2533\% remanescente após 360 dias (MINISTRY OF HEALTH, 2019); tempo de meia-vida nos sedimentos de 205 dias; mas somente 6,1 dias na fase aquática (PPDB, 2019); a $25^{\circ} \mathrm{C}$, em qualquer valor de $\mathrm{pH}$, não há hidrólise significativa.

Diante de vários estudos realizados com a Azoxistrobina e a grande quantidade de vegetais alvos da aplicação deste fungicida, é importante respeitar a época correta de aplicação, momento em que a cultura alvo não esteja no período de florescimento, pois parte dos vegetais são utilizados por abelhas, inclusive as do gênero Bombus, para coleta de alimentos, ficando expostos a tal produto.

\section{Acetamiprido}

O acetamiprido é um inseticida neonicotinoide de primeira geração, sistêmico que atua por contacto ou ingestão. Seu ingrediente ativo é o acetamiprido (acetamiprid). É um produto para aplicação foliar de diversas culturas. Dentre os neonicotinoides, destacam-se os produtos, imidacloprido, acetamiprido e tiametoxam. Estes inseticidas são agonistas dos receptores nicotínicos da acetilcolina (nAChR) presentes no sistema nervoso central dos insetos. Em estudos comparativos, verificou-se que os neonicotinoides apresentam grande afinidade aos $\mathrm{nAChR}$ de insetos do que de vertebrados, sendo menos tóxicos ao homem, portanto, empregados no manejo de insetos-praga (TOMIZAWA et al., 2005), tornando-se um dos principais grupos de produtos fitossanitários comercializado no mundo (SPARKS et al., 2015).

Os neonicotinoides geralmente são tóxicos para os insetos em pequenas quantidades quando comparado com outros grupos de inseticidas. Por exemplo, a dose letal 50 (DL50) para a ingestão de imidacloprido e clotianidina para abelhas é 5 e $4 \mathrm{ng}$ por insetos, respectivamente, o que para efeitos de comparação é de aproximadamente $1 / 10.000$ da DL50 para diclorodifeniltricloroetano (SUCHAIL et al., 2000).

De acordo com Takahashi et al. (1992), acetamiprido parece ser mais seguro para as abelhas do gênero Apis do que Bombus, porém, de uma maneira geral, é considerado mais seguro para as abelhas que os demais representantes dos neonicotinoides. A variação existente é devida, principalmente, à estrutura química dos produtos fitossanitários desse grupo e a afinidade pelos seus respectivos receptores nas abelhas. Por exemplo, os neonicotinoides podem ser divididos em dois grupos: (i) um formado por aqueles que possuem o radical $\mathrm{N}$-nitroguanidina (imidacloprido, tiametoxam, clotianidina e dinotefuram) e (ii) outro com 
Determinação de resíduos de agrotóxicos em Bombus spp. (Hymenoptera: Apidae) empregando o método Quechers modificado e LC-MS/MS e

radical $\mathrm{N}$-ciano-amidina (acetamiprido e tiacloprido). Em ambos os casos, esses inseticidas são agonistas do nAChR (ELBERT et al., 2008).

Estudos comparativos entre abelha e moscas mostraram que, em todos os casos, a afinidade do receptor da acetilcolina com acetamiprido é semelhante, sugerindo que a baixa susceptibilidade das abelhas não está relacionada com diferenças nos sítios de ação desses compostos (IWASA et al., 2004). Por outro lado, avaliando somente Apis mellifera (LINNEAUS, 1758), Iwasa et al. (2004) verificaram que tiametoxam chega a ser 192 vezes mais tóxicos que acetamiprido e tiacloprido, sendo essa variação atribuída ao fator estrutural da molécula e no caso do acetamiprido, durante o processo de metabolismo "in vivo" não gerar compostos tóxicos como ocorre com tiametoxam e imidacloprido (BRUNET et al., 2005).

Porém, de acordo com Pereira (2010), o acetamiprido aplicado nas doses que correspondem à DL50 e DL50/10, prejudicou o comportamento de reflexo e extensão da probóscide de $A$. mellifera nos testes realizados 1 e 4 horas após a aplicação do ingrediente ativo, enquanto que, a dose correspondente à DL50 prejudicou a atividade locomotora nesses mesmos períodos, e a dose correspondente à DL50/10 também afetou a atividade locomotora no teste de 4 horas.

\section{Metomil}

O metomil é um inseticida e acaricida, altamente tóxico para seres humanos, gado, animais de estimação e animais selvagens. Possui como ingrediente ativo o metomil (methomyl) e grupo químico: metilcarbamato de oxima. É um produto para aplicação foliar nas culturas de algodão, arroz, aveia, batata, brócolis, café, centeio, cevada, couve, dendê, feijão, milheto, milho, repolho, soja, sorgo, tomate, trigo e triticale. E para aplicação pré-plantio nas culturas de milho e soja. Este agrotóxico possui classificação toxicológica classe I. Observa-se que o risco dos agrotóxicos metomil, carbaril, cloropirifós, diazinona, dimetoato, ometoato, fentiona, metamidofós, metidationa, monocrotofós para as abelhas é mais elevado para aquelas que procuram alimento até 10 horas depois da pulverização $(E I C H, 2015)$. Segundo ValdovinosNúnezet et al. (2009), efeitos subletais de doses de metomil e permetrina (0,90 g p.c./ L de H2O e 0,91 g p.c./ L de $\mathrm{H} 2 \mathrm{O}$, respectivamente), demonstraram alta suscetibilidade para abelhas indígenas.

De acordo com Pimentel et al. (2012) as quantidades de inseticidas aplicadas muitas vezes não atingem o inseto alvo e boa parte do que é aplicado é perdido por deriva, o que aumenta ainda mais problemas com intoxicações a longas distâncias por insetos não alvos. Se tratando de A. mellifera, segundo Thompson et al. (2007), a exposição a doses subletais de inseticidas pode ter uma influência sobre a aprendizagem do inseto, sua capacidade de orientação, forrageamento e na sua prole.

Assim como a azoxistrobina (fungicida sistêmico), que é utilizado para proteger diversos vegetais do ataque de doenças, o acetamiprido (inseticida do grupo dos neonicotinoides) e o metomil (inseticida e acaricida), também são utilizados para proteger uma grande quantidade de vegetais da ação de insetospragas e ácaros, e suas aplicações tem que ser evitadas no período de floração, período de maior atividade das abelhas. É importante respeitar as normas para a aplicação de agrotóxicos e o período correto de 
Determinação de resíduos de agrotóxicos em Bombus spp. (Hymenoptera: Apidae) empregando o método Quechers modificado e LC-MS/MS e GC-MS/MS

aplicação. Realizando a utilização de agrotóxicos com segurança, as vidas dos insetos benéficos, assim como a dos seres humanos e animais, estarão mais preservadas.

No entanto, para Viana et al. (2010) a maior suspeita é que os polinizadores vêm desaparecendo de áreas agrícolas por causa da fragmentação de habitats, grandes áreas de monocultura, desmatamento para intensificar a agricultura e/ou pastagem e, principalmente devido ao uso excessivo ou incorreto de agrotóxicos. E de acordo com os resultados obtidos nas análises de resíduos de agrotóxicos nas amostras de Bombus estudadas, a possível causa da redução populacional de Bombus no Rio Grande do Sul não esteja diretamente associada ao uso de agrotóxicos, visto a pouca quantidade de resíduos detectada nas amostras, contudo, estudos mais ampliados são necessários para apoiar tal afirmativa.

\section{CONCLUSÕES}

Dos 119 agrotóxicos analisados, apenas azoxistrobina, acetamiprido e metomil, apresentaram resíduos detectáveis nas amostras analisadas, no entanto, a azoxistrobina foi detectada na concentração abaixo do limite de quantificação do método (LOQ) para amostra de B. morio coletados no município de Júlio de Castilho, enquanto que, foram detectados resíduos de acetamiprido e metomil acima do limite de quantificação do método em amostras também de B. morio, coletados nos municípios de Santiago e Capão do Cipó. Nos demais compostos (116), a concentração de agrotóxico obtida foi menor que o limite de detecção do método (LOD). Com isso, a suposta causa da redução populacional ou desaparecimento de abelhas do gênero Bombus no Rio Grande do Sul não esteja ligada diretamente a utilização de agrotóxicos nos plantios agrícolas do Estado, e sim pela expansão da agricultura e desmatamento que reduzem os locais propícios para nidificação, assim como os recursos florais utilizados por esses polinizadores.

\section{REFERÊNCIAS}

BADIOU-BÉNÉTEAU, A.; BENNEVEAU, A.; GÉRET, F.; DELATTE, H.; BECKER, N.; BRUNET, J. L.; REYNAUD, B.; BELZUNCES, L. P.. Honeybee biomarkers as promising tools to monitor environmental quality. Environ Int, v.60, p.31-41, 2013. DOI: http://doi.org/10.1016/i.envint.2013.07.002

BARTLETT, D.; CLOUGH, J.; GODWIN, J.; HALL, A.; HAMER, M.; PARR-DOBRZANSKI, B.. Review: the strobilurin fungicides. Pest Management Science, v.58, p.649-662, 2002.

BAYO, S. F.; GOKA, K.. Impacts of Pesticides on Honey Bees. Beekeeping and Bee Conservation, p.78-97, 2016.

BREEZE, T. D.; BAILEY, A. P.; BALCOMBE, K. G.; POTTS S. G.. Pollination services in the UK: How important are honeybees? Agr Ecosyst Environ, v.142, p.137-143, 2011.

BRUNET, J. L.; BADIOU, A.; BELZUNCES, L. P.. In vivo metabolic fate of [14C]- acetamiprid in six biological compartments of the honeybee, Apis mellifera Linneaus, 1758. Pest Management Science, Sussex, v.61, p.742-748, 2005.
COLLINS, C. H.; BRAGA, G. L.; BONATO, O. S.. Fundamentos de Cromatografia. 2 ed. Campinas: UNICAMP, 2009.

COLIN, M. E.; BELZUNCES, L. P.. Evidence of synergy between prochloraz and deltamethrin in Apis mellifera L. - A convenient biological approach. Pestic Sci, v.36, p.115-119, 1998.

EICH, A. P. P.. Histórico de uso e ação de agroquímicos sobre abelhas da espécie Apis mellifera. Monografia (Bacharelado em Ciências Biológicas) - Universidade Federal de Pampa, São Gabriel, 2015.

ELBERT, A.; HAA, M.; SPRINGER, B.; THIELERT, W.; NAUEN, R.. Applied aspects of neonicotinoid uses in crop protection. Pest Management Science, Sussex, v.64, p.1099-1105, 2008.

ELLIS, A.. Colony Collapse Disorder (CCD) in Honey Bees. Department of Entomology and Nematology, UF/IFAS Extension, 2007.

FERRER, I.; THURMAN, E. M. J.; CHROMATOGR A.. Multiresidue method for the analysis of 101 pesticides and their degradates in food and water samples by liquid 
chromatography/time-of-flight mass spectrometry. J

Chromatogr A, v.1175, n.1, p.24-37, 2007. DOI:

http://doi.org/10.1016/j.chroma.2007.09.092

GRADISH, A. E.; SCOTT-DUPREE, C. D.; FREWIN, A. J.; CUTLER, $G$. C.. Lethal and sublethal effects of some insecticides recommended for wild blueberry on the pollinator Bombus impatiens. The Canadian Entomologist, v.144, n.3, p.478486, 2012.

HARRIS, D. C.. Análise Química Quantitativa. 8 ed. Rio de Janeiro: LTC, 2012.

IWASA, T.; MOTOYAMA, N.; AMBROSE, J. T.; ROE, R. M.. Mechanism for the differential toxicity of neonicotinoid insecticides in the honey bee, Apis mellifera. Crop Protection, Guildford, v.23, p.371-378, 2004.

MALASPINA, O.; SOUZA, T. F.; SILVA-ZACARIN, E. C. M.; CRUZ, A. S.; JESUS, D.. Efeitos provocados por agrotóxicos em abelhas no Brasil. In: ENCONTRO SOBRE ABELHAS. Anais. Ribeirão Preto: FUNPEC, 2008. p.41-48.

MINISTRY OF HEALTH. Guidelines for drinking-water quality management for New Zealand (2nd edn). Datasheets. Part 2.3. Chemical and physical determinands. Pesticides. Wellington: Ministry of Health, 2019.

PARADIS, D.; BÉRAIL, G.; BONMATIN, J. M.; BELZUNCES, L. P. Sensitive analytical methods for 22 relevant insecticides of 3 chemical families in honey by GC-MS/MS and LC-MS/MS. Anal Bioanal Chem, v.406, p.621-633, 2014.

PEREIRA, A. M.. Efeitos de inseticidas na sobrevivência e no comportamento de abelhas. Tese (Doutorado em Ciências Biológicas) - Universidade Estadual Paulista "Júlio de Mesquita Filho", Rio Claro, 2010.

PIMENTEL, D.; BURGESS, M.. Small amounts of Pesticides Reaching Target Insects. Environment, Developement and Sustainability, v.14, p.1-2, 2012.

POTTS, S. G.; ROBERTS, S. P. M.; DEAN, R.; MARRIS, G.; BROWN, M. A.; JONES, R.; NEUMANN, P.; SETTELE, J.. Declines of managed honey bees and beekeepers in Europe.

J Apic Res, v.49, p.15-22, 2010. DOI:

http://doi.org/10.3896/ibra.1.49.1.02

PPDB. Pesticide Properties Database. Azoxystrobin. University of Hertfordshire, 2019.

SAIBT, N.. Determinação Multirresíduo de Agrotóxicos em Abelha e Pólen Apícola Empregando Método Quechers Modificado e LC-MS/MS. Dissertação (Mestrado em Química) - Universidade Federal de Santa Maria, Santa Maria, 2017.
SPARKS, T. C.; NAUEN, R.. IRAC: Mode of action classification and insecticide resistance management. Pesticide Biochemistry and Physiology, San Diego, v.121, p.122-128, 2015.

SUCHAIL, S. G.; GUEZ, D.; BELZUNCES, L. P.. Characteristics of imidacloprid toxicity in two Apis mellifera subspecies. Enviromental Toxicology Chemistry, v.19, p.1901-1905 2000.

TAKAHASHI, H.; MITSUI, J.; TAKAKUSA, N.; MASTUDA, M.; YONEDA, H.; SUZUKI, J.; ISHIMITSU, K.; KISHIMOTO, T.. NI25, a new type of systemic and broad spectrum insecticide. Brighton Crop Protection Conference, Brighton, UK. Pests and Diseases, v.1, 1992.

THOMPSON, H. M.; MAUS, C.. The Relevance of Sublethal Effects in Honey Bee Testing for Pesticide Risk Assessment. Pest Management Science, v.63, p.1058-1061, 2007.

TOMIZAWA, M.; CASIDA, J. E.. Neonicotinoid insecticide toxicology: mechanisms of Selective Action. Annual Review of Pharmacology and Toxicology, Palo Alto, v.45, p.247-268, 2005. DOI:

http://doi.org/10.1146/annurev.pharmtox.45.120403.09593 $\underline{0}$

VALDOVINOS-NÚNEZ, G. R.. Comparative Toxicity of Pesticides to Stingless Bess (Hymenoptera: Apidae: Meliponine). Journal of Economic Entomology, Gainesville, v.102, n.5, p.1737-1742, 2009.

VAN DER SLUIJS, J. P.; SIMON-DELSO, N.; GOULSON, D.; MAXIM, L.; BONMATIN, J. M.; BELZUNCES, L. P.. Neonicotinoids, bee disorders and the sustainability of pollinator services. Curr Opin Environ Sustain, v.5, n.3-4, p.293-305, 2013. DOI:

https://doi.org/10.1016/i.cosust.2013.05.007

VANENGELSDORP, D.; EVANS, J. D.; SAEGERMAN, C.; MULLIN, C.; HAUBRUGE, E.; NGUYEN, B. K.; FRAZIERM FRAZIER, J.; COX-FOSTER, D.; UNDERWOOD, R. C.; TARPY, D. R.; PETTIS, J. S.. Colony Collapse Disorder: A Descriptive Study. PLoS One, v.4, n.8, 2009.

http://doi.org/10.1371/journal.pone.0006481

VEIGA, M. M.. Análise da contaminação dos sistemas híbridos por agrotóxicos numa pequena comunidade rural do sudeste do Brasil. Caderno Saúde Pública, Rio de Janeiro, v.22, p.2391-2399, 2006.

VIANA, B. F.; SILVA, F. O.. Polinização por abelhas em agroecossistemas. SEBRAE, 2010.

ZANELLA, R.; PRESTES, O. D.; ADAIME, M. B.; MARTINS, M. L.. Preparo de amostras para análise de compostos orgânicos. Rio de Janeiro: LTC, 2015.

A CBPC - Companhia Brasileira de Produção Científica (CNPJ: 11.221.422/0001-03) detém os direitos materiais desta publicação. Os direitos referem-se à publicação do trabalho em qualquer parte do mundo, incluindo os direitos às renovações, expansões e disseminações da contribuição, bem como outros direitos subsidiários. Todos os trabalhos publicados eletronicamente poderão posteriormente ser publicados em coletâneas impressas sob coordenação da Sustenere Publishing, da Companhia Brasileira de Produção Científica e seus parceiros autorizados. Os (as) autores (as) preservam os direitos autorais, mas não têm permissão para a publicação da contribuição em outro meio, impresso ou digital, em português ou em tradução. 